\title{
Flutter Analysis of Piezoelectric Material Based Smart Wind Turbine Blade
}

\author{
Hao Wang, Junyu Yi, Wei Chen and Zhexin Zhou \\ College of Energy and Mechanical Engineering, Shanghai University of Electric Power, Shanghai, China. \\ E-mail:whouco@163.com
}

(Received 3 March 2021; accepted 18 June 2021)

\begin{abstract}
This paper presents a smart wind turbine blade of piezoelectric material. Based on Theodorsen unsteady aerodynamics and the V-g method, the flutter analysis in frequency domain is carried out for the smart wind turbine blade and the ordinary wind turbine blade. The simulation results demonstrate that the flutter critical velocity, that is, the reduced velocity of the smart wind turbine blade, is obviously much higher than that of the ordinary wind turbine blade. The smart wind turbine blade of piezoelectric material can effectively restrain the flutter of the wind turbine blade, especially for the flap motion. For the torsion motion, the smart wind turbine blade is kept away from the critical flutter. Then, to investigate the influences of different parameters on the flutter of the smart wind turbine blade, the influences of the center of gravity, the frequency ratio and the mass ratio of the blades on the flutter critical velocity of the smart wind turbine blade are researched respectively. The increase of the applied external electrical load of the piezoelectric material can increase the flutter critical velocity of the smart wind turbine blade.
\end{abstract}

\section{INTRODUCTION}

Under the background of the global environmental pollution and the shortage of fossil fuel energy, wind energy, as a type of green and renewable energy, has been paid special attention to by many countries. Therefore, the promotion and utilization of wind power generation is a universal trend in the world. As the core component of capturing wind energy, the wind turbine blade is getting larger and larger. Due to the increased flexibility of modern multi-megawatt wind turbine structures, more advanced analyses are needed to investigate the effects of geometric nonlinearities originating from large blade deformations under operational loads. ${ }^{1}$ The aeroelastic coupling flutter is undoubtedly the most common and the most dangerous for modern large-scale flexible wind turbine blades. The flutter of the blade is often caused by the coupling deformation of rotating bending torsion for the large space flexible blade, and the coupling of aerodynamic and elastic deformation. ${ }^{2}$

Once wind turbine blade flutter occurs, it will cause large amplitude vibration and make the blade break in a very short period of time. This leads to extremely serious consequences for the wind turbine blade. Research on the blade flutter problem, on the one hand, is helpful to understand and reveal the various aeroelastic phenomena occurrence regularity and mutual transformation of the conditions and mechanism. Research also provides a theoretical basis of the optimization design of the blade. On the other hand, it is necessary to prevent and eliminate the aeroelastic instability, and to lay the necessary foundation for the study of reducing the fatigue damage caused by the flutter of the blade structure. As a result, flutter analysis has been a very important factor in the design of wind turbines. Ahasan et al. theoretically proved the absolute possibility of generating electricity from the wind turbine blades' vibration and natural frequency while using piezoelectric material as raw material for turbine blade construction. ${ }^{3}$ Shakya et al. presented the flutter prediction of a composite wind turbine blades in the time domain aeroelastic analysis using unsteady blade element momentum (BEM) theory as aerodynamic model. ${ }^{4}$ With the development of new types of smart materials, the piezoelectric materials with the characteristics of sensing and actuation have attracted the attention of many researchers. ${ }^{5}$ A new solution was proposed by Elahi et al., to extract energy from Limit Cycle Oscillation (LCO) phenomenon through the use of the energy conversion of the piezoelectric materials. ${ }^{6}$ Otiefy and Negm applied the finite element method of piezoelectric self-sensing actuator to research the flutter suppression ability of the wing. ${ }^{7}$ Kashfi et al. proposed the feasibility of using piezoelectric actuators as a smart material for vibration suppression of wind turbine blades. ${ }^{8}$ Qiao et al. studied the active control characteristics of the wind turbine blade vibration. ${ }^{9}$ Azadi et al. applied piezoelectric electrode as actuators to suppress the vibration of the attachment. ${ }^{10}$ Liu analyzed the classical flutter of the blade based on the piezoelectric drive, but did not give the degree of inhibition of piezoelectric materials on the blade flutter. ${ }^{11}$ Huang et al. explored the flutter instability mechanism of large blades and analyzed the influence of aerodynamic damping on flutter. ${ }^{12}$ Piezoelectric materials have been widely used in the suppression of aircraft wings flutter, but as for the wind turbines, it is still in the initial stage.

This paper focuses on the flutter analysis of the ordinary wind turbine blade and the wind turbine blade of piezoelectric material. And it is organized as follows: flutter problem of two element airfoils based on Theodorsen unsteady aerodynamic force, flutter analysis of two kinds of wind turbine blades of the V-g method, influence of parameters on the flutter critical velocity of wind turbine blade of piezoelectric materials and conclusions in the end.

\section{FLUTTER PROBLEM OF TWO ELEMENT AIRFOIL BASED ON THEODORSEN UNSTEADY AERODYNAMIC FORCE}

\subsection{The Equation of Motion of Two Degrees of Freedom Wind Turbine Airfoil}

Two elements airfoil has only two or three degrees of freedom, so the blade flutter can be dealt with in the physical space. 


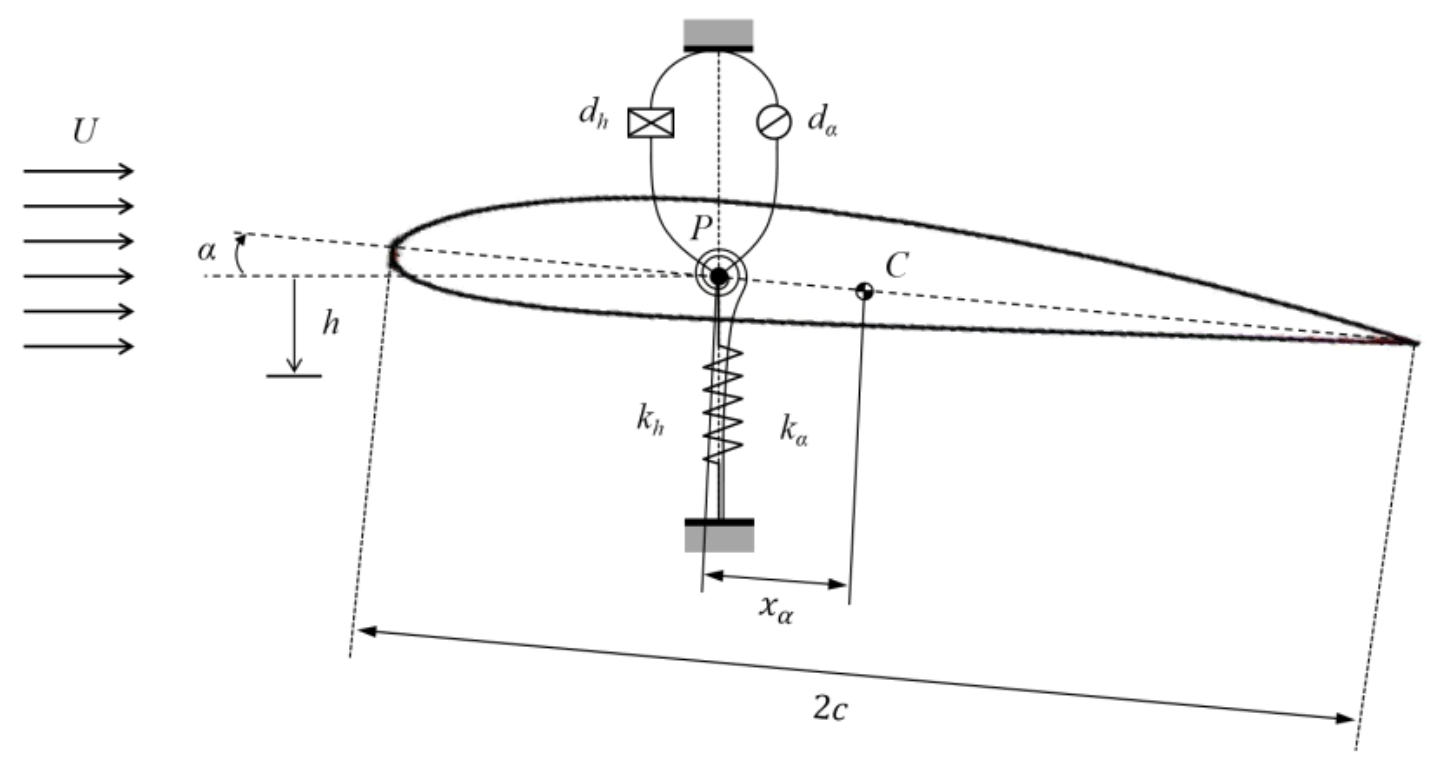

Figure 1. Aerodynamic airfoil section of a common blade under uniform airflow.

It is not necessary to transform the structural dynamic equation into the modal space. An airfoil with two degrees of freedom is taken into account, as shown in Fig. 1.

The cross section was applied by the uniform incompressible potential flow, whose airflow velocity was $U .^{13} P$ was the reference point of the airfoil motion. $C$ was the center of mass. The stiffness coefficients of the flapping and torsion directions of the reference point $P$ were $k_{h}$ and $k_{\alpha}$ respectively. The damping coefficients were $d_{h}$ and $d_{\alpha}$ respectively. The flap motion and torsion motion were $h$ and $\alpha$ respectively. The $c$ was the half chord length, and $x_{\alpha}$ was the distance between center of mass and center of stiffness.

According to Fig. 1, the equation of motion for two elements airfoil was given as Eq. (1):

$$
\left[\begin{array}{cc}
m & m x_{\alpha} \\
m x_{\alpha} & I_{\alpha}
\end{array}\right]\left[\begin{array}{c}
\ddot{h} \\
\ddot{\alpha}
\end{array}\right]+\left[\begin{array}{cc}
k_{h} & 0 \\
0 & k_{\alpha}
\end{array}\right]\left[\begin{array}{l}
h \\
\alpha
\end{array}\right]=\left[\begin{array}{c}
F \\
M_{\alpha}
\end{array}\right]
$$

where $m$ was the mass of blade per unit length, $F$ was the aerodynamic force, $M_{\alpha}$ was the aerodynamic moment, and $I_{\alpha}$ was the moment of inertia.

Equation (1) can also be changed into Eq. (2) as follows:

$$
\left[\begin{array}{cc}
1 & \bar{x}_{\alpha} \\
\bar{x}_{\alpha} & \bar{r}_{\alpha}^{2}
\end{array}\right]\left[\begin{array}{c}
\ddot{h} / c \\
\ddot{\alpha}
\end{array}\right]+\left[\begin{array}{cc}
\omega_{h}^{2} & 0 \\
0 & \omega_{\alpha}^{2} \bar{r}_{\alpha}^{2}
\end{array}\right]\left[\begin{array}{c}
h / c \\
\alpha
\end{array}\right]=\left[\begin{array}{c}
F /(m c) \\
M_{\alpha} /\left(m c^{2}\right)
\end{array}\right] ;
$$

where $\omega_{\alpha}=\sqrt{k_{\alpha} / I_{\alpha}}$ was the torsion natural frequency, $\omega_{h}=$ $\sqrt{k_{h} / m}$ was the flap natural frequency, $\bar{x}_{\alpha}=x_{\alpha} / c$ was the ratio of the distance between the center of mass and the center of stiffness to the half chord length, $\bar{r}_{\alpha}=r_{\alpha} / c$ was the ratio of the radius of gyration of blade around center of stiffness to the half chord length, $r_{\alpha}$ was the radius of gyration of blade around the center of stiffness, and the definition of the other symbols were shown as before.

If the speed of the blade reached the flutter speed, the wind turbine blade moved harmonically as Eq. (3)

$$
h=\bar{h} e^{i \omega t} ; \quad \alpha=\bar{\alpha} e^{i \omega t} .
$$

As a result, the aerodynamic forces and aerodynamic moments changed harmonically

$$
F=\bar{F} e^{i \omega t} ; \quad M_{\alpha}=\bar{M}_{\alpha} e^{i \omega t} .
$$

When substituting Eqs. (3) and (4) into Eq. (2), it gave rise to Eq. (5)

$$
\begin{array}{r}
-\omega^{2}\left[\begin{array}{cc}
1 & \bar{x}_{\alpha} \\
\bar{x}_{\alpha} & \bar{r}_{\alpha}^{2}
\end{array}\right]\left[\begin{array}{c}
h / c \\
\alpha
\end{array}\right]+\left[\begin{array}{cc}
\omega_{h}^{2} & 0 \\
0 & \omega_{\alpha}^{2} \bar{r}_{\alpha}^{2}
\end{array}\right]\left[\begin{array}{c}
h / c \\
\alpha
\end{array}\right]= \\
\\
{\left[\begin{array}{c}
F \\
\bar{M}_{\alpha} /(m c) \\
\bar{M}_{\alpha} /(m)^{2}
\end{array}\right] .}
\end{array}
$$

According to the Theodorsen theory, the unsteady aerodynamic forces and moments were given as follows

$$
\begin{aligned}
\bar{F}= & \pi \rho_{a} c^{3} \omega^{2}\left\{\frac{\bar{h}}{c} L_{h}+\left[L_{\alpha}-\left(\frac{1}{2}+\bar{a}\right) L_{h}\right] \bar{a}\right\} \\
\bar{M}_{\alpha}= & \pi \rho_{a} c^{4} \omega^{2}\left\{\left[M_{h}-\left(\frac{1}{2}+\bar{a}\right) L_{h}\right] \frac{\bar{h}}{c}+\right. \\
& {\left.\left[M_{\alpha}-\left(\frac{1}{2}+\bar{a}\right)\left(L_{\alpha}+M_{h}\right)+\left(\frac{1}{2}+\bar{a}\right)^{2} L_{h}\right] \bar{a}\right\} ; }
\end{aligned}
$$

where the four complex coefficients of blade for harmonic vibration were given as Eq. (8)

$$
\begin{aligned}
L_{h} & =1-i 2 C(k) \frac{1}{k} ; & L_{\alpha} & =\frac{1}{2}-i \frac{1+2 C(k)}{k}-\frac{2 C(k)}{k^{2}} ; \\
M_{h} & =\frac{1}{2} ; & M_{\alpha} & =\frac{3}{8}-i \frac{1}{k} ;
\end{aligned}
$$

where $C(k)$ was the Theodorsen function.

Therefore, considering the parameters in Eqs. (6)-(8), Eq. (5) was described as Eq. (9)

$$
-\Omega^{2}[\mathbf{M}]\left[\begin{array}{c}
\bar{h} / c \\
\bar{\alpha}
\end{array}\right]+[\mathbf{K}]\left[\begin{array}{c}
\bar{h} / c \\
\bar{\alpha}
\end{array}\right]=\Omega^{2}[\mathbf{A}(k)]\left[\begin{array}{c}
\bar{h} / c \\
\bar{\alpha}
\end{array}\right]
$$

where $\quad \Omega^{2}=\omega^{2} / \omega_{\alpha}^{2} ; \quad R^{2}=\omega_{h}^{2} / \omega_{\alpha}^{2} ;$

$$
\begin{aligned}
& {[\mathbf{M}]=\left[\begin{array}{cc}
1 & \bar{x}_{\alpha} \\
\bar{x}_{\alpha} & \bar{r}_{\alpha}^{2}
\end{array}\right] ; \quad[\mathbf{K}]=\left[\begin{array}{cc}
R_{\omega}^{2} & 0 \\
0 & \bar{r}_{\alpha}^{2}
\end{array}\right] ; \quad[\mathbf{A}(k)]=} \\
& {\left[\begin{array}{cc}
L_{h} & L_{\alpha}-\left(\frac{1}{2}+\bar{a}\right) L_{h} \\
M_{h}-\left(\frac{1}{2}+\bar{a}\right) L_{h} & M_{\alpha}-\left(\frac{1}{2}+\bar{a}\right)\left(L_{\alpha}+M_{h}\right)+\left(\frac{1}{2}+\bar{a}\right)^{2} L_{h}
\end{array}\right] .}
\end{aligned}
$$




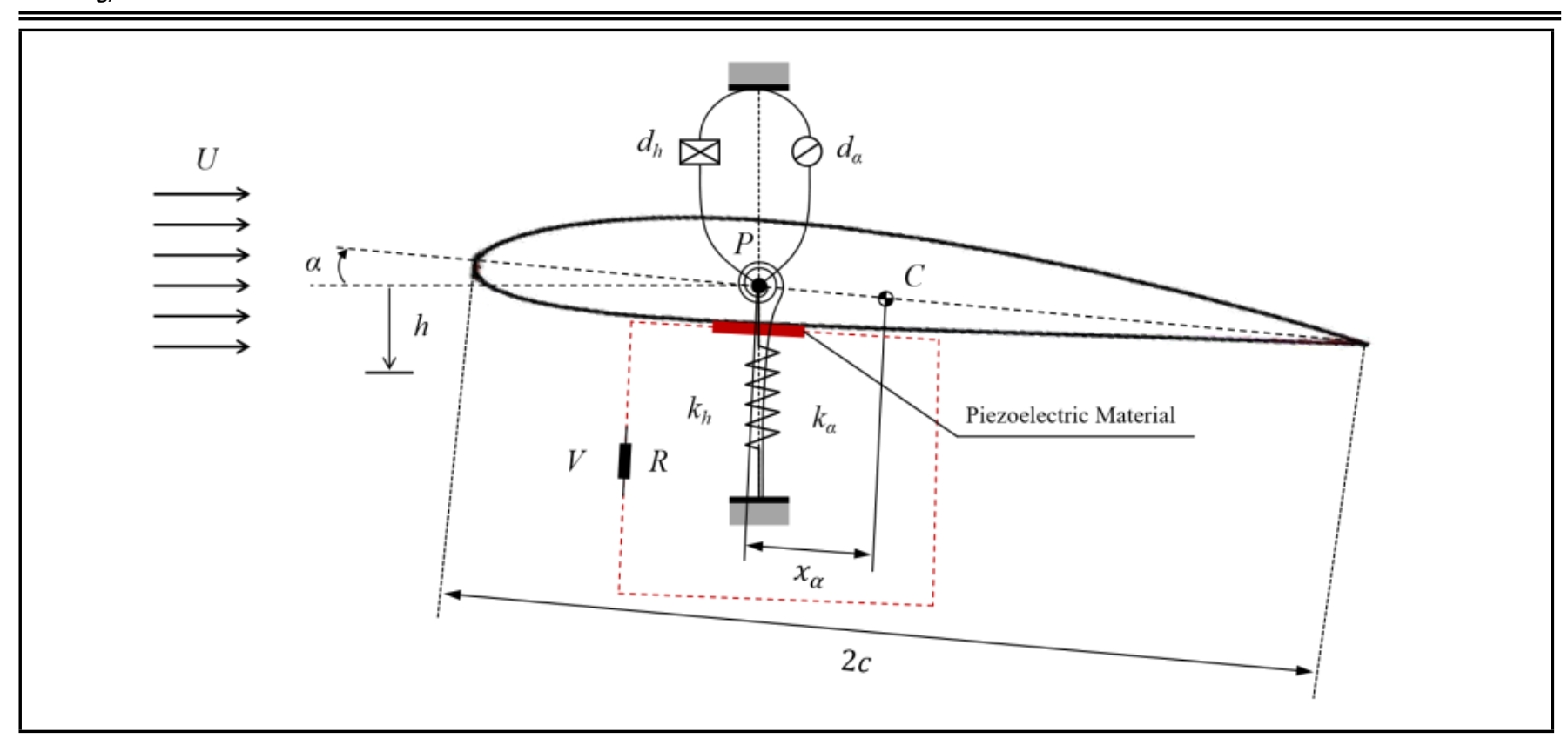

Figure 2. Aeroelastic airfoil section of piezoelectric material under uniform airflow.

\subsection{The Equation of Motion of the Two Degrees of Freedom Wind Turbine Airfoil with Piezoelectric Coupling}

The aeroelastic airfoil section of piezoelectric material under uniform airflow is shown in the Fig. 2, which was similar with that of the common blade shown in Fig. 1. The piezoelectric cross section was governed by the incompressible potential flow. ${ }^{14}$ In addition, the flapping direction satisfied the piezoelectric coupling, and the voltage generated by the aeroelastic wind turbine blade system was connected to the external electrical load resistor $R, V$ was the voltage of $R$.

For the wind turbine blade system with piezoelectric material, the Lagrange method was used to establish the equation of motion through the use of kinetic energy, potential energy and internal electric energy as Eqs. (10)-(12).

(1) Total kinetic energy per unit length:

$$
T=\frac{1}{2} m \dot{h}^{2}+x_{\alpha} c \dot{h} \dot{\alpha}+\frac{1}{2} m_{f} \dot{h}^{2}+\frac{1}{2} I_{\alpha} \dot{\alpha}^{2} ;
$$

where $m_{f}$ meant the quality of the connecting fixture $\left(m_{f}=0\right.$ in ideal condition), $I_{\alpha}$ denoted the moment of inertia of reference point.

(2) Total potential energy per unit length:

$$
U=\frac{1}{2} k_{h} h^{2}+\frac{1}{2} k_{\alpha} \alpha^{2}-\frac{1}{2} \frac{\theta}{l} h v
$$

where $\theta$ was the electromechanical coupling term, $l$ was the long span and $v$ was the output voltage.

(3) Internal electric energy per unit length:

$$
W_{i e}=\frac{1}{2} \frac{C_{p}^{e p}}{l} v^{2}+\frac{1}{2} \frac{\theta}{l} h v
$$

where $C_{p}^{e p}$ was the equivalent capacitance of piezoelectric ceramic layer.

The positive direction of aerodynamic $F$ was downward, and the aerodynamic moment $M_{\alpha}$ of around center of stiffness was positive when heading up in the wind. The kinetic equation, the potential energy equation and the internal energy equation is introduced into the Lagrange equation, that is, Eq. (13) $)^{15}$

$$
\begin{aligned}
& \frac{d}{d t}\left(\frac{\partial T}{\partial \dot{h}}\right)-\frac{\partial T}{\partial h}+\frac{\partial U}{\partial h}-\frac{\partial W_{i e}}{\partial h}+d_{h} \dot{h}=-L \\
& \frac{d}{d t}\left(\frac{\partial T}{\partial \dot{\alpha}}\right)-\frac{\partial T}{\partial \alpha}+\frac{\partial U}{\partial \alpha}-\frac{\partial W_{i e}}{\partial \alpha}+d_{\alpha} \dot{\alpha}=M \\
& \frac{d}{d t}\left(\frac{\partial T}{\partial \dot{v}}\right)-\frac{\partial T}{\partial v}+\frac{\partial U}{\partial v}-\frac{\partial W_{i e}}{\partial v}=\frac{Q}{l}
\end{aligned}
$$

where $Q$ was the output electric charge.

Therefore, the equation of motion of the smart wind turbine blade of piezoelectric material can be descripted as Eq. $(14)^{15}$

$$
\begin{aligned}
& \left(m+m_{f}\right) \ddot{h}+m x_{\alpha} b \ddot{\alpha}+d_{h} \dot{h}+k_{h} h-\frac{\theta}{l} v=L \\
& m x_{\alpha} b \ddot{\alpha}+I_{\alpha} \ddot{\alpha}+d_{\alpha} \dot{\alpha}+k_{\alpha} \alpha=N \\
& C_{p}^{e p} \dot{v}+\frac{v}{R}+\theta \dot{h}=0
\end{aligned}
$$

where $R$ was the external electrical load resistor (the unit is $\Omega$ ).

\section{FLUTTER ANALYSIS OF TWO KINDS OF WIND TURBINE BLADES WITH V-G METHOD}

\subsection{Flutter Analysis of the Ordinary Blade}

The V-g method was used to analyze the flutter of wind turbine blade in frequency domain, based on the Theodorsen unsteady aerodynamic force. The flutter problem can be solved using the complex eigenvalues of the resonant aerodynamic forces. The real and imaginary parts of complex eigenvalues were solved respectively. ${ }^{16}$ Introducing a parameter $g$, the critical point of wind turbine blade flutter was obtained by an effective graph method, that is V-g graph.

Firstly, the structural damping of the system was assumed to be zero, then artificial structural damping was introduced, the 
equation of motion is shown as Eq (15)

$$
\left[\begin{array}{cc}
m & m x_{\alpha} \\
m x_{\alpha} & I_{\alpha}
\end{array}\right]\left[\begin{array}{l}
\ddot{h} \\
\ddot{\alpha}
\end{array}\right]+\left[\begin{array}{cc}
k_{h} & 0 \\
0 & k_{\alpha}
\end{array}\right]\left[\begin{array}{l}
h \\
\alpha
\end{array}\right]=\left[\begin{array}{c}
F \\
M_{\alpha}
\end{array}\right]+\left[\begin{array}{c}
D_{h} \\
D_{\alpha}
\end{array}\right]
$$

where $D_{h}=i g m \omega_{h}^{2} \bar{h} e^{i \omega t}, D_{\alpha}=i g I_{\alpha} \omega_{\alpha}^{2} \bar{h} e^{i \omega t}$ meant the dissipation structural damping force.

Substituting the structural damping force into Eq. (15), then a generalized eigenvalue problem was produced as Eq. $(16)^{17}$

$$
([\mathbf{A}(k)]+[\mathbf{M}])\left\{\begin{array}{c}
\bar{h} / c \\
\bar{\alpha}
\end{array}\right\}=\frac{(I+i g)}{\Omega^{2}}[\mathbf{K}]\left\{\begin{array}{c}
\bar{h} / c \\
\bar{\alpha}
\end{array}\right\} .
$$

The characteristic value of Eq. (16) can be described as Eq. (17)

$$
\lambda=\frac{(1+i g)}{\Omega^{2}}=\lambda_{\mathrm{Re}}+i \lambda_{\mathrm{Im}}
$$

and, $\omega=\omega_{\alpha} / \sqrt{\lambda_{\operatorname{Re}}}, g=\lambda_{\operatorname{Im}} / \lambda_{\operatorname{Re}}$ was the introduced artificial structural damping coefficients.

Considering the reduced frequency $k=(\omega c) / V$, so $V=$ $\left(\omega_{\alpha} C\right) / k \sqrt{\lambda_{\mathrm{Re}}}$. When $g$ was zero, the imaginary part of the eigenvalue $\lambda_{\operatorname{Re}}$ was zero. The conditions of flutter were met, so $V$ was the flutter speed at this time.

The calculating procedure of the flutter analysis using the $\mathrm{V}-\mathrm{g}$ method can be shown as follows:

1. Decide the region of reduced frequency of interest.

2. Calculate the general aerodynamic matrix $A(k)$ and solve eigenvalues $\lambda$.

3. Determine the $g, \omega, V$ at the current reduced frequency.

4. Repeat the above procedures at the new reduced frequency according to the step size decided.

5. Plot the V-g curve consequently.

To be simple, the dimensionless reduced velocity $V_{\text {non }}=$ $V / \omega_{\alpha} c$ was used to replace the flutter critical velocity in the following part.

\subsubsection{Parameters of Ordinary Blade for Flutter Analysis}

Taking a $1.5 \mathrm{MW}$ wind turbine blade as an example, the critical velocity of the blade was predicted using the V-g method. The basic parameters of the blade were as follows: $\bar{x}_{\alpha}=0.025, \bar{r}_{\alpha}=0.2549, m=185 \mathrm{~kg}, \omega_{\alpha}=16.103$, $R_{\omega}=0.0419, c=0.5365, \bar{\alpha}=-0.1073, k_{\alpha}=3117 \mathrm{Nm} / \mathrm{rad}$, $k_{h}=11.429 \mathrm{~N} / \mathrm{m}, \rho_{\alpha}=1.225 \mathrm{~kg} / \mathrm{m}^{3}$.

According to the relationship between $V_{\text {non }}$ and $g$, the point where $g$ was zero in the graph was the critical point of the flutter.

\subsubsection{Simulation Result and Analysis}

Figure 3 shows the relationship between the reduced velocity and the $g . g$ was zero when the $V_{\text {non }}$ was equal to about 3.70 . It means that when $V_{\text {non }}=3.70$, the blades fluttered in flap direction. For the angular displacement, the flutter will not occur at all, because the V-g graph for the angular displacement had no intersection with the line of $g=0$.

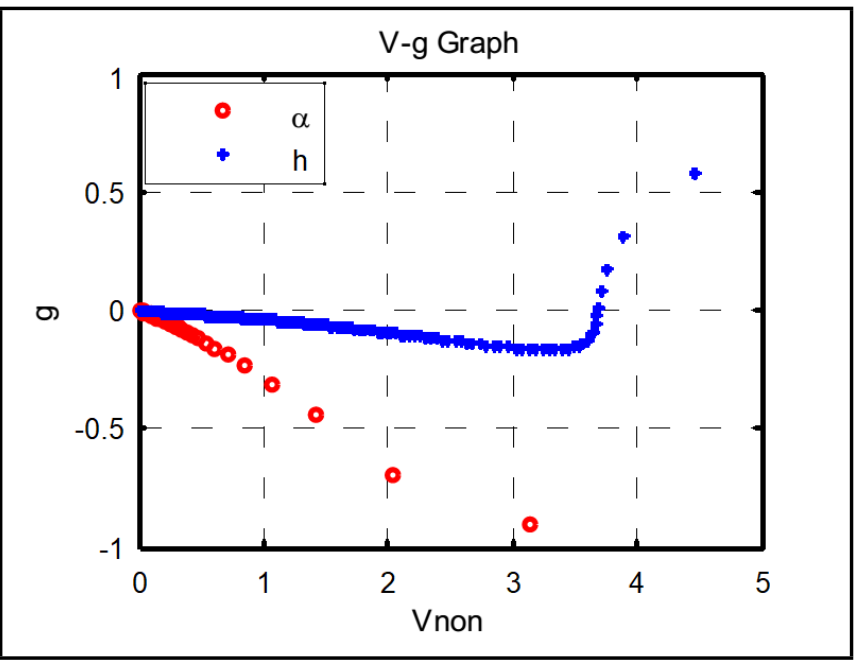

Figure 3. The V-g graph for the ordinary wind turbine blade.

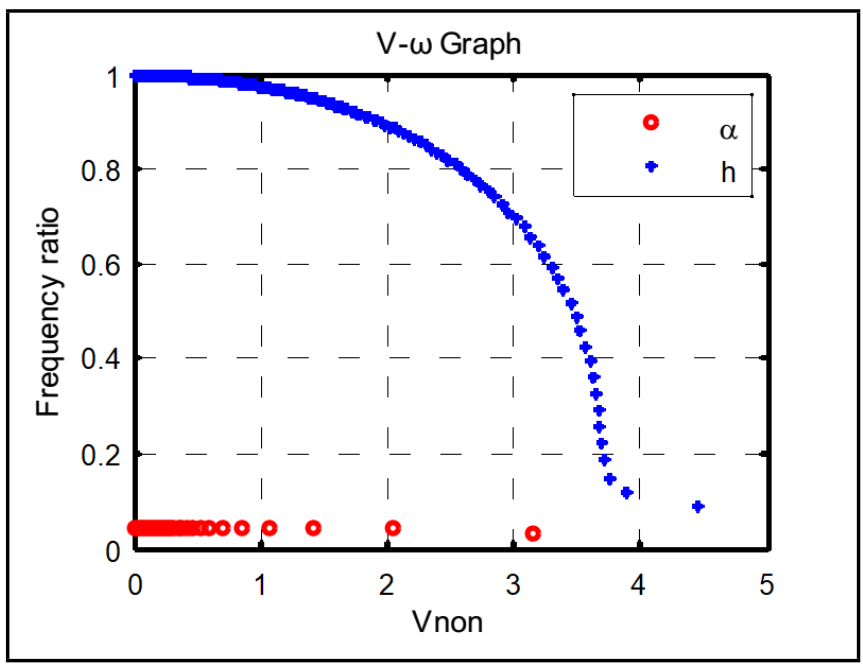

Figure 4. The V- $\omega$ graph for the ordinary wind turbine blade.

\subsection{Flutter Analysis of Blade with Piezoelectric Materials}

The V-g method was also used to analyze the smart wind turbine blade with piezoelectric materials. When the wind turbine blade reached the flutter critical velocity, the wind turbine blade with piezoelectric material moved harmonically. Therefore, the following assumptions were obtained: $h=\bar{h} e^{i \omega t}$, $\alpha=\bar{\alpha} e^{i \omega t}, v=\bar{v} e^{i \omega t}, L=\bar{L} e^{i \omega t}, N=\bar{N} e^{i \omega t}$.

Therefore, for the flap motion and torsion motion, the eigenvalue problem can be shown as Eq. (18)

$$
\begin{aligned}
& {\left[\begin{array}{cc}
\beta+\frac{l_{h}}{\mu}-\kappa(\omega)-\sigma^{2}\left(1+i \gamma_{h}\right) \lambda & x_{\alpha}+\frac{l_{\alpha}}{\mu} \\
x_{\alpha}+\frac{m_{\alpha}}{\mu} & r^{2}+\frac{m_{\alpha}}{\mu}-r^{2}\left(1+i \gamma_{\alpha}\right) \lambda
\end{array}\right] .} \\
& \left\{\begin{array}{c}
\bar{h} / c \\
\bar{\alpha}
\end{array}\right\}=\left[\begin{array}{l}
0 \\
0
\end{array}\right]
\end{aligned}
$$

where $\lambda=\left(\omega_{\alpha} / \omega\right)^{2}$ was the complex eigenvalue, $\sigma=\omega_{h} / \omega_{\alpha}$ was the frequency ratio, $r=\sqrt{I_{p} / m c^{2}}$ was the radius of gyration, $\mu=m / \pi \rho_{\infty} c^{2}$ was the mass ratio of blade to air, $\gamma_{h}=\omega d_{h} / k_{h}, \gamma_{\alpha}=\omega d_{\alpha} / k_{\alpha}$ were the loss factors. $\beta=\left(m+m_{f}\right) / m$ was the ratio of blade mass to the mass of the spring clamp. As $m_{f}$ was always equal to zero, $\beta$ was 
often treated as 1. $l_{h}, l_{\alpha}, m_{h}, m_{\alpha}$ are shown as Eq. (19)

$$
\begin{aligned}
l_{h}= & 1-i 2 C(k) \frac{1}{k} \\
l_{\alpha}= & -a-i \frac{1}{k}-\frac{1}{2}-\frac{2 C(k)}{k^{2}}-i \frac{2}{k}\left(\frac{1}{2}-a\right) C(k) \\
m_{h}= & -a+i \frac{2}{k}\left(\frac{1}{2}+a\right) C(k) \\
m_{\alpha}= & \frac{1}{8}+a^{2}-i \frac{1}{k}\left(\frac{1}{2}-a\right)+\frac{2 C(k)}{k^{2}}\left(\frac{1}{2}+a\right)+ \\
& i \frac{2}{k}\left(\frac{1}{4}-a^{2}\right) C(k)
\end{aligned}
$$

where $a$ was the dimensionless position of the position of the reference point relative to the midpoint of chord length.

Assuming that the damping is not added to the system model, then $d_{\alpha}=0, d_{h}=0$. The damping correlation term was 0 , so the above eigenvalue equation can be written as follows

$$
\begin{gathered}
\left\{\left[\begin{array}{cc}
1 & \bar{x}_{\alpha} \\
\bar{x}_{\alpha} & \bar{r}_{\alpha}^{2}
\end{array}\right]-\left[\begin{array}{cc}
R_{\omega}^{2} & 0 \\
0 & \bar{r}_{\alpha}^{2}
\end{array}\right]+\left[\begin{array}{cc}
l_{h}-\mu \kappa(\omega) & l_{\alpha} \\
m_{h} & m_{\alpha}
\end{array}\right]\right\} \\
\left\{\begin{array}{c}
\ddot{h} / c \\
\bar{\alpha}
\end{array}\right\}=\left[\begin{array}{l}
0 \\
0
\end{array}\right]
\end{gathered}
$$

where $\kappa(\omega)=\frac{\theta^{2} i}{\omega m l\left(i \omega C_{p}^{e q}+1 / R_{1}\right)}$ was a frequency function obtained by eliminating the voltage term, and it depended on the complex eigenvalue $\lambda$. lem

Then Eq. (20) was treated as a generalized eigenvalue prob-

$$
-\Omega^{2}[\mathbf{M}]\left\{\begin{array}{c}
\bar{h} / c \\
\bar{\alpha}
\end{array}\right\}+[\mathbf{K}]\left\{\begin{array}{c}
\bar{h} / c \\
\bar{\alpha}
\end{array}\right\}=\Omega^{2}\left[\mathbf{A}^{\prime}(k)\right]\left\{\begin{array}{c}
\bar{h} / c \\
\bar{\alpha}
\end{array}\right\} ;
$$

where $\left[\mathbf{A}^{\prime}(k)\right]=\frac{1}{\mu}$.

$$
\left[\begin{array}{cc}
L_{h}-\mu \kappa(\omega) & L_{\alpha}-\left(\frac{1}{2}+\bar{a}\right) L_{h} \\
M_{h}-\left(\frac{1}{2}+\bar{a}\right) L_{h} & M_{\alpha}-\left(\frac{1}{2}+\bar{a}\right)\left(L_{\alpha}+M_{h}\right)+\left(\frac{1}{2}+\bar{a}\right)^{2} L_{h}
\end{array}\right] .
$$

\subsubsection{Example of Piezoelectric Material Blade}

The above 1.5 MW wind turbine blade was still used for the smart wind turbine blade. The basic parameters of the blade were the same as the above examples. The same analysis method was used to predict the flutter critical speed of the blade. Pasting the piezoelectric ceramic PZT-5A (QP10N, Mide) patch on the blade, therein electromechanical coupling term $\theta=1.55 \mathrm{mN} / \mathrm{V},{ }^{15}$ the equivalent capacitance $C_{p}^{e q}=$ $120 \mathrm{nF}$, the electrical load resistance $R=100000 \Omega$. Using $\mathrm{V}$-g method, the simulation results can be seen as follows.

\subsubsection{Analysis of the Effect of Piezoelectric Material on Flutter Velocity}

To investigate the V-g graph and the V- $\omega$ graph for the flap motion and the torsion motion with the ordinary wind turbine blade and the piezoelectric material blade, the same parameters were applied for these two kinds of wind turbine blades. In addition, the $\mathrm{V}-\mathrm{g}$ graphs for the flap motion and the torsion motion were considered respectively, and so does for the V- $\omega$ graph.

Figure 5 shows the comparison of the V-g graph for the flap motion with two different wind turbine blades. It can be found

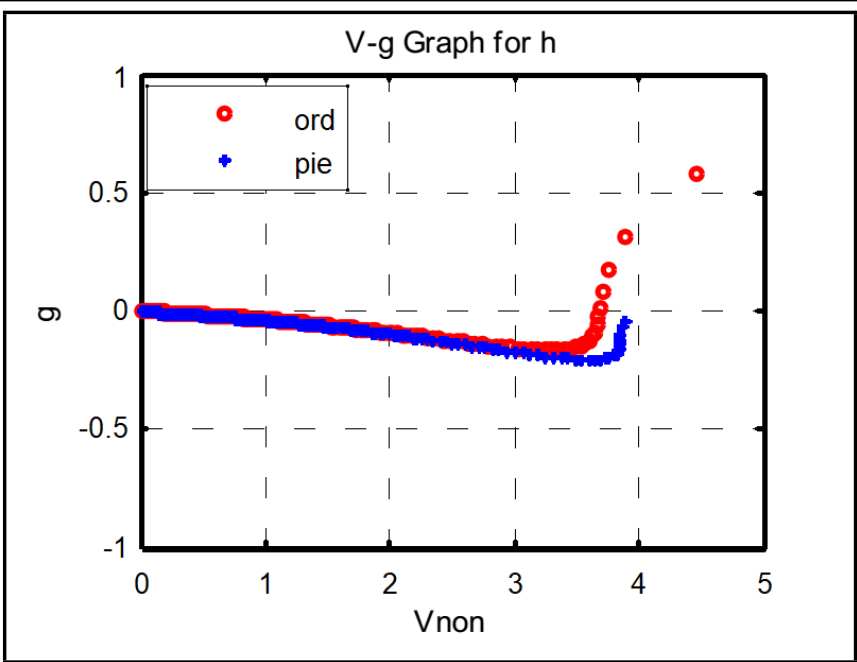

Figure 5. The V-g graph for flap motion of ordinary blade and piezoelectric material blade.

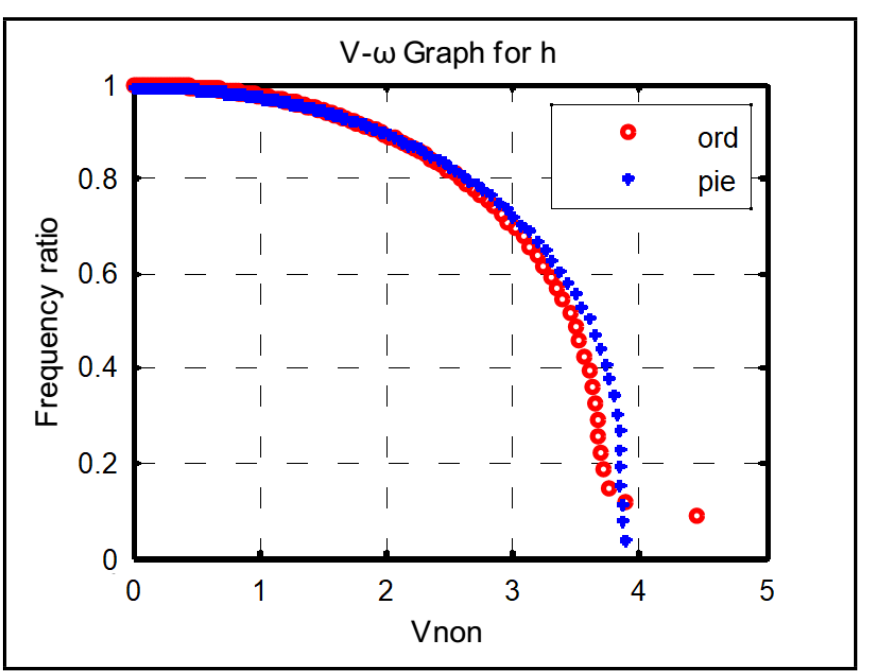

Figure 6. The V- $\omega$ graph for flap motion of ordinary blade and piezoelectric material blade.

that when the reduced velocity $V_{\text {non }}$ was infinitely close to 3.9 , $g=0$. For the blade of piezoelectric materials, it means that when $V_{\text {non }}$ was infinitely close to 3.9 , the blades could flutter.

Therefore, for the flap motion of the wind turbine blade, the reduced velocity of piezoelectric material one was $5.4 \%$ higher than that of the ordinary blade. And the flutter critical velocity of the piezoelectric materials blade was $5.4 \%$ higher than that of the ordinary blade. As for the blade of the same parameters, the increase in the flutter critical speed means that the possibility of the blade flutter was reduced to the extent. That is to say, adding the piezoelectric material into the wind turbine blade improved the flutter critical velocity of the blade, and restrained the occurrence of flutter effectively.

Figure 6 shows the comparison of the V- $\omega$ graph for the flap motion with two different wind turbine blades. It can be found that when $V_{\text {non }}$ was equal to about 3.9, the frequency ratio $\omega / \omega_{h}=0.3$, so the flutter frequency $\omega_{F}=0.3 \omega_{h}$. For the piezoelectric materials blade and ordinary blade, when $g=0$, the frequency ratio of the piezoelectric material blade was larger than that of the ordinary blade. The results show that, with the same parameters, the flutter frequency of the piezoelectric material blade is higher than that of the ordinary blades. 


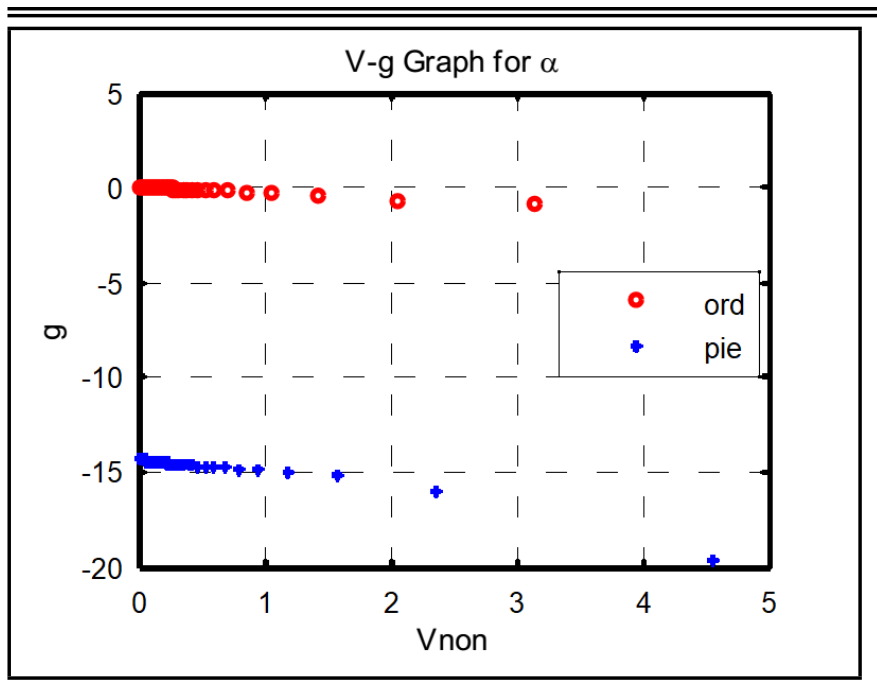

Figure 7. The V-g graph for torsion motion of ordinary blade and piezoelectric material blade.

Figure 7 shows the comparison of the V-g graph for the torsion motion with two different wind turbine blades. It can be shown that the V-g graph for the torsion motion could not intersect with the line which $g$ equals zero. Therefore, the flutter did not occur to the torsion motions of these two kinds of wind turbine blades. What's more, the possibility of flutter occurs to the torsion motion of the wind turbine blade with piezoelectric material was much less than that of the ordinary wind turbine blade. Because the V-g graph of the ordinary blade stays just near the line which $g$ equals zero, while the V-g graph of the piezoelectric one stays near the line where $g$ equals -15 .

Adding the piezoelectric material into the wind turbine blade will keep the V-g graph away from the line which $g$ equals zero, so the torsion motion was kept away from the flutter effectively.

Figure 8 shows the comparison of the V- $\omega$ graph for the torsion motion with two different wind turbine blades. It can be found that frequency ratio keeps almost unchanged for these two different wind turbine blades.

\section{INFLUENCE OF PARAMETERS ON THE FLUTTER CRITICAL VELOCITY OF WIND TURBINE BLADE OF PIEZOELECTRIC MATERIALS}

Considering three parameters including the position of gravity, frequency ratio, mass ratio, which had great influence on the blade performance, the V-g method was used to investigate the flutter analysis of wind turbine blade of piezoelectric material. By changing the value of the applied electrical load, its influence on flutter critical velocity of wind turbine blade with piezoelectric material blade will also be discussed. In the simulation process, the formal parameters of $1.5 \mathrm{MW}$ wind turbine mentioned above were used one more time.

\subsection{The Influence of Center of Gravity on the Flutter Critical Velocity}

In order to investigate the influence of center of gravity on the flutter critical velocity, all the parameters except the center of gravity were kept unchanged. The value of the center of gravity of blade changed between -0.15 and 0.45 . Under the

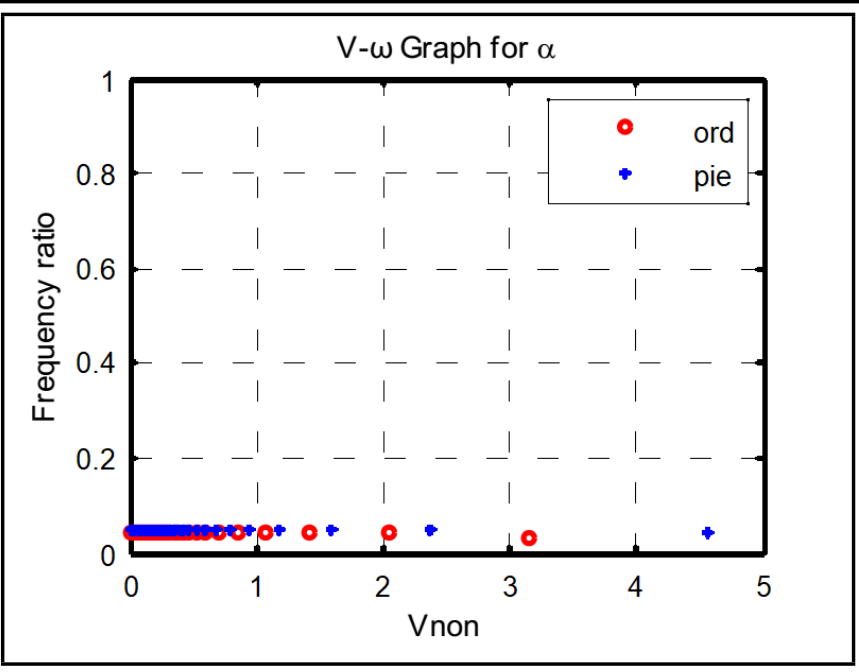

Figure 8. The V- $\omega$ graph for torsion motion of ordinary blade and piezoelectric material blade.

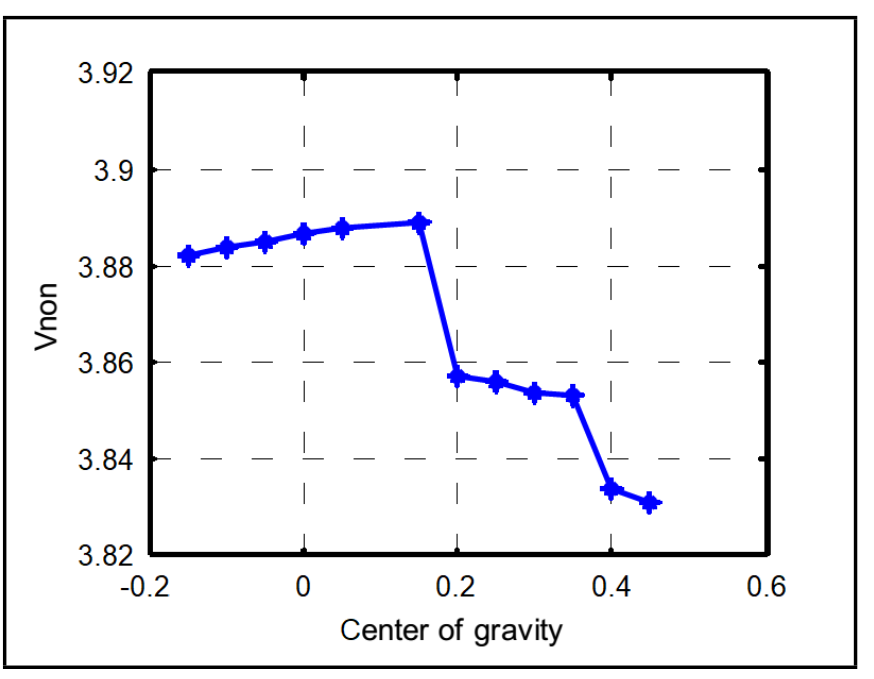

Figure 9. Relation between the center of gravity and the reduced velocity.

different positions of the center of gravity of blade, the change of the flutter critical velocity of blade is discussed. Each point of the reduced velocity from the figures like Fig. 5 was extracted to get Fig. 9.

It was shown that with the increasing of the position of the center of gravity, the critical flutter velocity demonstrates a gradually decreasing process. It is not difficult to find that, when $\bar{x}_{\alpha}=-0.15 \sim 0.15$, the critical flutter velocity stays near at 3.88. The critical flutter velocity shows a downward trend, but the degree of decline was quite slight. Therefore, for the piezoelectric material blade, the influence of the center of gravity on the critical flutter speed was not very significant. This is to say, the flutter critical velocity of the wind turbine blade of piezoelectric material was very stable when changing the position of center of gravity.

\subsection{Effect of Frequency Ratio on the Critical Flutter Velocity}

To investigate the influence of the frequency ratio on the flutter critical velocity, all the parameters except the frequency ratio were kept unchanged. The value of the frequency ratio was defined between 0.2 and 2.0. The changing law of flutter critical velocity is discussed considering different value of 


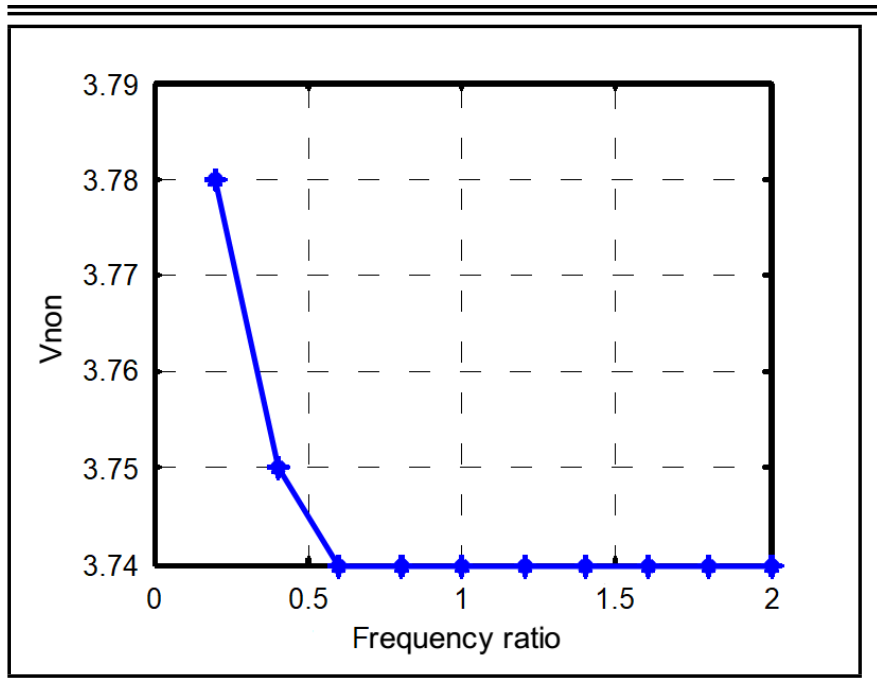

Figure 10. Relation between the frequency ratio and the reduced velocity.

frequency ratio, as shown in Fig. 10. And from Fig. 10, when the frequency ratio was less than 0.6 , the flutter critical velocity decreases, but the decreasing degree was very small. When the frequency ratio was larger than 0.6 , with the changing of frequency ratio, the flutter critical velocity stayed near at 3.74 . Therefore, the flutter critical velocity of wind turbine blade of piezoelectric material remained stable when changing the frequency ratio.

\subsection{Effect of Mass Ratio on the Flutter Critical Velocity}

To investigate the influence of the mass ratio on the flutter critical velocity, all the parameters except the mass ratio were kept unchanged. The flutter critical velocity of wind turbine blade of piezoelectric material is discussed under different values of the mass ratio between 30 and 230, as shown in Fig. 11. It is not difficult to see from Fig. 11 that with the increase of mass ratio, the flutter critical velocity gradually increases. The results show that it is not easy to cause flutter in the condition of small air density when the piezoelectric material is added into wind turbine blade.

\subsection{The Influence of Applied Electrical Load on the Critical Flutter Velocity}

In order to investigate the influence of the applied electrical load on flutter critical velocity, all the parameters except the applied electrical load were kept unchanged. The value of applied electrical load changed between $1 \Omega$ and $10000 \Omega$. Then the flutter critical velocity is discussed for the wind turbine blade of piezoelectric material, as shown in Fig. 12. From the simulation results, it can be seen that when the applied electrical load is less than $1000 \Omega$, the flutter critical velocity stays near at 3.7. When the value of applied electrical load changes between $1000 \Omega$ and $9000 \Omega$, with the increase of the $R$, the flutter critical velocity gradually increases. When the applied electrical load reached a certain value, the flutter critical velocity is unchanged. When applied electrical load reaches near $9000 \Omega$, the flutter critical velocity will reach the maximum value which is 3.94 .

Therefore, with the increase of the applied electrical load value, the flutter critical velocity of the wind turbine blade with piezoelectric material increases. That is to say, the flutter

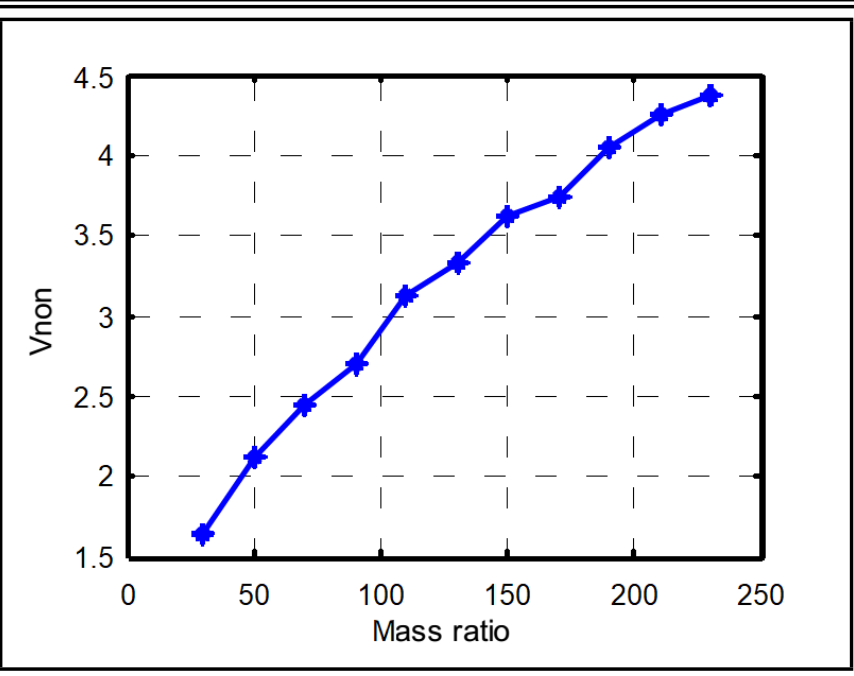

Figure 11. Relation between the mass ratio and the reduced velocity.

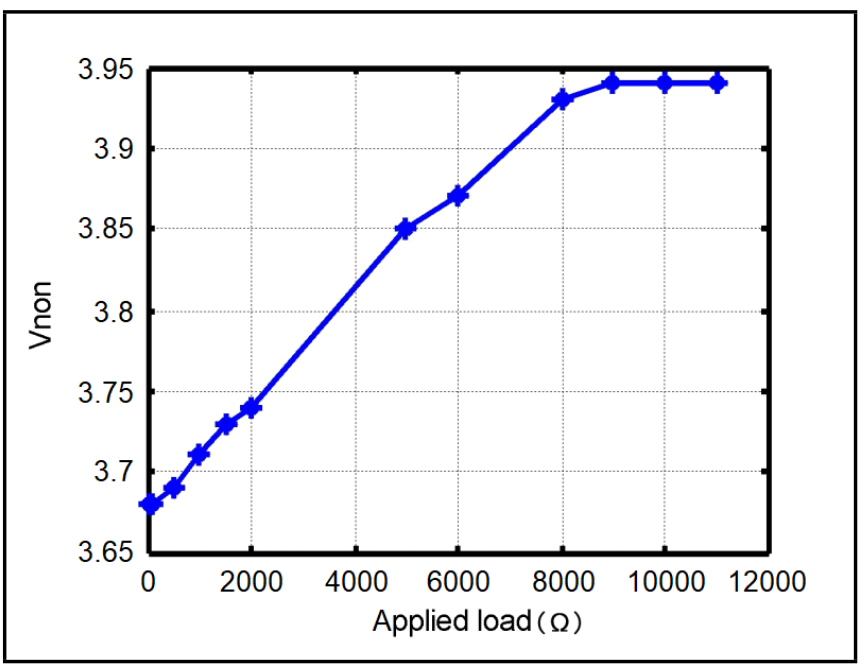

Figure 12. Relation between the applied electrical load and the reduced velocity.

of wind turbine blades of piezoelectric material are effectively suppressed.

\section{CONCLUSIONS}

(1) The equations of motion of two-dimensional airfoil are established for the flutter analysis of the ordinary wind turbine blade and the wind turbine blade of piezoelectric material respectively. Through the use of the V-g method of flutter analysis under the same conditions for two kinds of blade, the flutter critical velocity of the wind turbine blade of piezoelectric material is higher than that of the ordinary blade. The wind turbine blade can increase the flutter critical velocity by adding piezoelectric material, and it will become more difficult for wind turbine blade to cause flutter.

(2) The position of the center of gravity, the frequency ratio, the mass ratio of the smart wind turbine blade, and the applied external electrical load, are applied to investigate the flutter critical velocity of wind turbine blade with piezoelectric material under different parameters. There is little influence on the flutter critical velocity of piezoelectric material blade for the center of gravity and the frequency ratio. When the mass ratio increases, the flutter critical velocity will increase, so the piezoelectric material blade is not easy to flutter in the place 
with low air density. The applied external electrical load related to the piezoelectric material will increase the flutter critical velocity of wind turbine blade of piezoelectric material in a reasonable range.

(3) It is proven theoretically that the addition of piezoelectric material to the wind turbine blade can effectively restrain the occurrence of flutter. Because it is very difficult to add the piezoelectric materials effectively and conveniently into the wind turbine blade, there will be a long way to go in the future.

\section{ACKNOWLEDGEMENTS}

The work was supported by Capacity Building Projects in Local Universities of Science and Technology Commission of Shanghai Municipality (NO.19020500900).

\section{REFERENCES}

1 Rezaei, M. M., Zohoor, H., and Haddadpour, H. Aeroelastic modeling and dynamic analysis of a wind turbine rotor by considering geometric nonlinearities, Journal of Sound and Vibration, 432 (18), 653-679, (2018). https://dx.doi.org/10.1016/j.jsv.2018.06.063

2 Sayed, M., Lutz, Th., Krämer, E., Shayegan, Sh., and Wüchner, R. Aeroelastic analysis of $10 \mathrm{MW}$ wind turbine using CFD-CSD explicit FSI-coupling approach, Journal of Fluids and Structures, 87, 355-356, (2019). https://dx.doi.org/10.1016/j.jfluidstructs.2019.03.023

3 Ahasan, Md. F., Hassan, M. F., and Mondal, D. Numerical analysis of energy harvesting on a wind turbine blade by using piezoelectric material, International Conference on Mechanical, Industrial and Energy Engineering, 18, 2-6, (2018). Retrieved from https://www2. kuet.ac.bd/icmiee2018/files/ICMIEE18-273.pdf (Accessed March, 2021).

4 Shakya, P., Sunny, M. R., and Maiti, D. K. Time domain flutter analysis of bend-twist coupled large composite wind turbine blades: a parametric study, Mechanics Based Design of Structures and Machines, 207, 764-775, (2020). https://dx.doi.org/10.1080/15397734.2020.1824796

5 Bryant, M. and Garcia, E. Development of an aeroelastic vibration power harvester, The International Symposium On: Smart Structures and Materials \& Nondestructive Evaluation and Health Monitoring, 7288, 1-12, (2009). https://dx.doi.org/10.1117/12.815785

6 Elahi, H., Eugeni, M., Fune, F., Lampani, L., Mastroddi, F., Paolo Romano, G., and Gaudenzi, P. Performance evaluation of a piezoelectric energy harvester based on flag-flutter, Micromachines, 11 (10), 933, (2020). https://dx.doi.org/10.3390/mi11100933
7 Otiefy, R. A. H. and Negm, H. M. Wing box transonic-flutter suppression using piezoelectric selfsensing diagonal-link actuators, International Journal of Solids \& Structures, 48 (1), 31-43, (2011). https://dx.doi.org/10.1016/j.ijsolstr.2010.09.005

8 Kashfi, M., Fakhri, P., Amini, B., and Yavari, N. Vibration analysis of a wind turbine blade integrated by a piezoelectric layer, Proc. of 2019 International Power System Conference (PSC), 650-654, (2019). https://dx.doi.org/10.1109/PSC49016.2019.9081522

9 Qiao, Y., Han, J., Zhang, C., Chen, J., and Gou, R. Active vibration control of wind turbine blades by piezoelectric materials, Chinese Journal of Applied Mechanics, 30 (4), 586-591, (2013). https://dx.doi.org/10.11776/cjam.30.04.C074

10 Azadi, M., Azadi, E., and Fazelzadeh, S. A. Robust inverse dynamic control of a maneuvering smart flexible satellite with piezoelectric layers, International Journal of Acoustics and Vibration, 22 (4), 456-461, (2017). https://dx.doi.org/10.20855/ijav.2017.22.4491

11 Liu, T. Classical flutter and active control of wind turbine blade based on piezoelectric actuation, Shock and Vibration, 2015, 292368, 4-13, (2015). https://dx.doi.org/10.1155/2015/292368

12 Huang, J., Xia, H., Li, D., and Guo, K. Nonlinear aeroelastic modal analysis of large wind turbine flexible blades, Journal of Mechanical Engineering, 56, 181-187, (2020). https://dx.doi.org/10.3901/JME.2020.14.180

13 Suatean, B., Galetuse, S., and Colidiuc, A. Aeroelastic problems of wind turbine blades, AIP Conference Proceeding, 1281, 1867-1870, (2010). https://dx.doi.org/10.1063/1.3498270

14 Han, J.-H., Tani, J., and Qiu, J. Active flutter suppression of a lifting surface using piezoelectric actuation and modern control theory, Journal of Sound and Vibration, 291 (3-5), 706-722, (2006). https://dx.doi.org/10.1016/j.jsv.2005.06.029

15 Erturk, A. and Inman, D. J. Piezoelectric Energy Harvesting, John Wiley \& Sons Ltd, UK, (2011).

16 Liu, N., Bai, J., Liu, Y., and Hua, J. Efficient flutter prediction based on harmonic balance and V-g methods, Acta Aerodynamica Sinica, 34 (5), 631-637, (2016). https://dx.doi.org/10.7638/kqdlxxb-2015.0178

17 Inoue, T., Ishida, Y., and Kiyohara, T. Nonlinear vibration analysis of the wind turbine blade (Occurrence of the superharmonic resonance in the out of plane vibration of the elastic blade), Journal of Vibration and Acoustics, 134 (13), 031009, (2012). https://dx.doi.org/10.1115/1.4005829 